\title{
Características psicométricas de un cuestionario en español para medir estrategias de aprendizaje del inglés como lengua extranjera
}

\author{
PILAR Franco \\ Universidad de Cádiz \\ Margarita PINO \\ Universidad de Vigo \\ BEATRIZ RODRÍGUEZ \\ Universidad Nacional de Educación a Distancia
}

Recibido: 18 de enero de 2011 / Aceptado: 5 de septiembre de 2012 ISSN: $1697-7467$

\begin{abstract}
RESUMEN: El objetivo de este estudio es diseñar, aplicar y validar un cuestionario como instrumento de medición para comprobar el uso de estrategias de aprendizaje en la enseñanza del inglés como lengua extranjera.

Está compuesto de ocho preguntas de identificación, 58 ítems cerrados con preguntas dicotómicas, lo que nos permite saber el porcentaje de individuos que utiliza una determinada estrategia, y una escala tipo Likert que permite conocer la frecuencia de uso de estrategias.

El análisis de los ítems se realizó mediante el índice de homogeneidad, el cálculo de la consistencia interna y la validez de constructo.

Palabras clave: Estrategias de aprendizaje, aprendizaje de lenguas extranjeras, instrumento de medición, análisis de factores, procesos de aprendizaje.
\end{abstract}

Psychometric properties of a Spanish version of a questionnaire about strategies for learning English as a foreign language

\footnotetext{
ABSTRACT: The aim of this study is to design, test and validate a questionnaire as a survey instrument to identify the use of strategies for learning English as a foreign language.

It consists of eight questions for students' identification, 58 close-ended questions for yes and no answers, which allow the analysis of the percentage of students who use a particular strategy, and a Likert-type scale for each of the 58 items that allows the students to report the frequency of strategy use.

The item analysis was performed using the homogeneity index, the calculation of internal consistency and construct validity.

Keywords: Learning strategies, foreign language learning, survey instrument, factor analysis, processes for learning.
} 


\section{Marco teórico}

El campo de la investigación sobre las estrategias para el aprendizaje de una lengua no materna requiere que se realicen estudios que aporten respuestas a dos de las críticas más importantes manifestadas hasta el momento. En primer lugar, la necesidad de un marco conceptual basado en las teorías del aprendizaje y del procesamiento de la información que aporte homogeneidad en la definición y clasificación de las estrategias. En segundo lugar, se requieren estudios que evalúen los instrumentos de medida así como los procedimientos de obtención y análisis de datos para contribuir a su refinamiento.

Por un lado, en la elaboración de los instrumentos de investigación así como en el análisis e interpretación de los datos se deberán tener en cuenta clasificaciones basadas en las teorías del aprendizaje y del procesamiento de la información. De esta forma, estas clasificaciones serán representativas del uso de las estrategias en estos procesos.

Por otro, los instrumentos de medida que se desarrollen deben depender del tipo de datos que se desea obtener para responder a las preguntas concretas de cada investigación en cuestión. Asimismo, la objetividad de los datos obtenidos debe ser puesta en cuestión y debe buscarse siempre una mejora en los sistemas de medición para tender a eliminar cualquier elemento subjetivo.

Respecto al primer punto, el diseño del presente cuestionario se basa en un modelo que explica los procesos que intervienen en el procesamiento de la información y el aprendizaje. Autores como Wenden (1991), O'Malley y Chamot (1990), Oxford (1990), Valcarcel, Coyle y Verdú (1996); Cohen (1998), Bruton (1998), Skehan (1998) en el campo del aprendizaje de las lenguas no maternas y Beltrán (1996) y Bernard (1999) en el ámbito del aprendizaje en general, han explicado los procesos que se producen durante el procesamiento de información y el aprendizaje siguiendo a grandes nombres de la psicología cognitiva y la lingüística aplicada como Anderson (1981, 1985), Hunt (1982), Fodor (1983), Gagné (1985), Schmidt (1990), VanPatten (1990, 1996) y Schumann (1994) entre otros.

En líneas generales casi todos los autores coinciden en establecer de algún u otro modo los siguientes procesos:

Tabla 1. Procesos que intervienen en el procesamiento de la información y el aprendizaje

\begin{tabular}{|l|l|}
\hline Proceso de sensibilización: & $\begin{array}{l}\text { Se refiere al contexto mental y afectivo del aprendizaje } \\
\text { donde juegan un papel muy importante la motivación, la } \\
\text { emoción y las actitudes (Beltrán, 1996). Aquí se destaca- } \\
\text { rían las estrategias afectivas y sociales. }\end{array}$ \\
\hline Proceso del input: & $\begin{array}{l}\text { La información que se obtendría del entorno, pasaría a } \\
\text { través de los receptores y se registraría a través de los } \\
\text { órganos sensoriales específicos para lo cual se emplea- } \\
\text { rían las estrategias cognitivas atencionales. A continua- } \\
\text { ción, la información se situaría en la memoria operativa o }\end{array}$ \\
\hline
\end{tabular}




\begin{tabular}{|l|l|}
\hline & $\begin{array}{l}\text { a corto plazo en donde las estrategias cognitivas de } \\
\text { representación y organización de los datos serían deci- } \\
\text { sivas. }\end{array}$ \\
\hline Proceso central: & $\begin{array}{l}\text { Consistiría en el almacenamiento de la información en la } \\
\text { memoria a largo plazo para lo que intervendrían también } \\
\text { las estrategias cognitivas de organización destacando } \\
\text { los procesos de deducción, inducción y extrapolación } \\
\text { de manera que el alumno alcance la comprensión y el } \\
\text { dominio del objeto de aprendizaje. }\end{array}$ \\
\hline Proceso del output: & $\begin{array}{l}\text { El alumno es capaz de utilizar los elementos de la memo- } \\
\text { ria a corto y largo plazo a través de las estrategias de } \\
\text { recuperación y de transferencia para dar respuestas } \\
\text { necesarias demandadas por el entorno donde se encuen- } \\
\text { tre. }\end{array}$ \\
\hline Proceso de evaluación: & $\begin{array}{l}\text { Tanto el alumno como el profesor comprueban el apren- } \\
\text { dizaje que se ha llevado a cabo para lo cual son impres- } \\
\text { cindibles las estrategias metacognitivas. }\end{array}$ \\
\hline
\end{tabular}

Teniendo en cuenta estos procesos, para el diseño del presente cuestionario, se ha adoptado el modelo representado en el Gráfico 1. En este gráfico se representan los distintos procesos que intervienen en el procesamiento de la información y del aprendizaje en relación con las estrategias de aprendizaje.

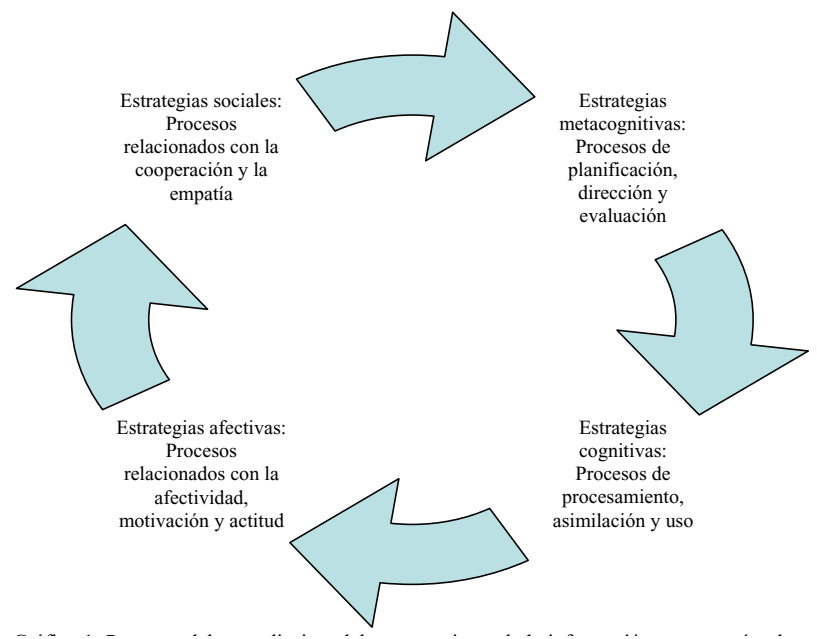

Gráfico 1. Procesos del aprendizaje y del procesamiento de la información y categorias de estrategias que intervienen en los mismos 
Los procesos representados no se producen de forma aislada sino que están relacionados entre sí y se influyen mutuamente. Así pues, las estrategias de aprendizaje juegan un papel muy importante en estos procesos, y son elementos indispensables para su desarrollo. Más adelante, en el apartado dedicado al instrumento de investigación se explicará con mayor detalle cómo estos procesos se desarrollan a través del uso de las estrategias.

Por tanto, para este estudio se ha diseñado un instrumento de investigación basado en el marco teórico presentado que explica cómo intervienen las estrategias de aprendizaje en los procesos de aprendizaje y del procesamiento de la información.

Asimismo, la presente investigación responde también a la necesidad, en el campo de las estrategias de aprendizaje, de evaluar el instrumento de medida elaborado así como los procedimientos de obtención y análisis de datos.

Respecto a las técnicas utilizadas hasta el momento, existen ya algunos trabajos que se han aproximado a los diferentes métodos de investigación sobre las estrategias de aprendizaje (White, Schramm y Chamot, 2007).

Igualmente, Oxford (1990,1996), Cohen, (1998), y Cohen y Macaro (2007) distinguen cinco tipos principales de técnicas (entrevistas, observaciones, reflexiones, anotaciones, diarios y cuestionarios) para la evaluación de estrategias de aprendizaje (EA) y señala que las técnicas más libres proporcionan mayor riqueza y gozan de más espontaneidad, pero los resultados son más difíciles de cuantificar y de interpretar de forma global. Sin embargo, los formatos más cerrados aportan datos que pueden resumirse y analizarse de forma más objetiva. Es por este motivo que el presente estudio se haya realizado para diseñar, aplicar y evaluar un instrumento de este tipo.

Como paso previo a la elaboración del cuestionario, se ha realizado una revisión bibliográfica de aquellos estudios más representativos respecto a este instrumento. Se han tenido en cuenta tanto aquéllos que analizan las estrategias de aprendizaje en general como las relacionadas con las destrezas o los contenidos lingüísticos.

Respecto a las que analizan el uso de EA sin relacionarlas con una tarea, destreza o contenido lingüístico, cabe destacar como uno de los primeros cuestionarios desarrollados y más utilizado es el Inventario de Estrategias para el Aprendizaje de una lengua (IEAL) traducido del inglés Strategy Inventory for language Learning (SILL) desarrollado por Oxford (1990) para evaluar el uso de las estrategias de aprendizaje en general. Las estrategias están agrupadas en seis apartados, siguiendo las categorías establecidas por Oxford (estrategias de memoria, cognitivas, de compensación, metacognitivas, afectivas y sociales). Existen dos versiones del IEAL (Oxford, 1990: 283-300): la versión 5.1, para hablantes de inglés que aprenden otra lengua, y la versión 7.0 (ESL/EFL) para hablantes de otras lenguas que aprenden inglés.

Igualmente, existen autores como Fernández (2004) que han realizado estudios basándose en una adaptación del cuestionario de Oxford (1990). En concreto, esta autora, realiza un estudio empírico con 160 estudiantes de cuatro niveles distintos de conocimiento del inglés como lengua extranjera (ILE) para analizar qué estrategias usan y con qué frecuencia dentro de cada grupo. Entre otros instrumentos utiliza un cuestionario que consta de dos partes, una primera sobre datos personales y una segunda que consiste en una adaptación del SILL. 
Después del IEAL, han aparecido cuestionarios que miden el uso de las estrategias para las destrezas y los contenidos lingüísticos conjuntamente como el Cuestionario de Estrategias de una Lengua (Language Strategies Survey -LSS-) (Cohen, Oxford y Chi, 2003). Este instrumento, posteriormente revisado, sirve para comprobar las estrategias de aprendizaje que emplean los alumnos en el desarrollo de las cuatro destrezas (comprensión y expresión oral y escrita), además del vocabulario y la traducción. Asimismo, se considera, junto con el Inventario de Estrategias para el Aprendizaje de Cultura (Strategies Inventory for Learning Culture -SILC-) fiable y válido para medir el uso de estas estrategias (Paige, Cohen and Shively, 2004).

Existen también instrumentos que se dedican a medir las estrategias de aprendizaje en relación con una sola destreza. En esta línea, cabe destacar el Cuestionario de Toma de Conciencia Metacognitiva para la Comprensión Oral para comprobar el uso de las estrategias metacognitivas en la comprensión oral (traducido del inglés Metacognitive Awareness Listening Questionnaire -MALQ-) (Vandergrift et al. 2006). Al igual que el cuestionario anterior, también basado en los distintos procesos que intervienen en la metacognición, se ha diseñado el Inventario de Estrategias Metacognitivas para la Comprensión Escrita (traducido del inglés Metacognitive Awareness of Reading Strategies Inventory -MARSI-) (Sheorey y Mokhatari, 2001). Tanto uno como el otro son instrumentos que han sido mejorados tras su aplicación y cuya fiabilidad y validez han sido demostradas. Por último, mencionar también el Inventario de Estrategias para la Comunicación Oral (Nakatani, 2005) que sirve para analizar las estrategias empleadas para desarrollar la interacción y de esta forma realizar un estudio sobre un aspecto aún no suficientemente investigado.

A la vista de las investigaciones realizadas hasta el momento, el diseño, aplicación y evaluación de los resultados de cuestionarios sobre las estrategias de aprendizaje de una lengua extranjera puede aportar conclusiones relevantes. En esta línea, aunque se pueden analizar las estrategias en relación con una tarea, destreza o contenido lingüístico, es interesante también adoptar un enfoque más general sobre los procesos de aprendizaje y del procesamiento de la información. De esta manera, se puede llegar a conclusiones que enriquezcan el conocimiento sobre el uso de las estrategias de aprendizaje tomando en consideración los distintos aspectos que influyen en su uso.

\section{MÉTODO}

\subsection{Participantes}

El estudio se ha desarrollado en el Centro Universitario de Idiomas a Distancia (CUID). El CUID es una unidad docente especializada, creada en el año 2000, y está adscrito funcionalmente a la Facultad de Filología de la Universidad Nacional de Enseñanza a Distancia.

$\mathrm{Su}$ finalidad es promover el aprendizaje de las lenguas españolas oficiales e idiomas extranjeros como enseñanzas no regladas en los Centros Asociados de la UNED. Con su creación se pretende dotar preferentemente a los estudiantes universitarios de los conocimientos lingüísticos necesarios para un adecuado desarrollo de sus estudios y profesiones futuras. 
La matrícula se ha ido incrementando con el tiempo de forma exponencial y en el curso académico 2007-2008 estuvieron matriculados 4800 estudiantes en 38 Centros Asociados.

La investigación se ha desarrollado con 257 estudiantes. El 56,3\% son mujeres y el $43,7 \%$ hombres. La media de edad es 40 años, siendo la mínima 20 y la máxima 65 . El 59\% cursan nivel elemental y $41 \%$ nivel avanzado y el $65 \%$ acuden habitualmente a tutorías mientras el 35\% no lo hacen. La mayoría es licenciado (41\%) o diplomado (29\%), mientras que bachillerato lo han cursado el 11, $8 \%$ y formación profesional el 12,6\%. Solamente el $1,6 \%$ es doctor.

Con respecto a las profesiones que ejercen, solamente el 3,9\% son estudiantes, la mayoría son funcionarios $(42,8 \%)$ : rama administrativa $(16,4 \%)$, educación $(13,8 \%)$, sanidad (12,6\%); administrativos no funcionarios (16, 8\%); construcción (ingeniero, aparejador, arquitecto) (8,3\%); sector servicios (turismo, comercio, transportista, hostelería, estilista) $(10,5 \%)$; ama de casa $(3,1 \%)$; jubilados $(2,6 \%)$; otros (sacerdote, artes escénicas, periodista, etc.) $(10,2 \%)$; no contesta $(1,9 \%)$.

Las razones para estudiar una segunda lengua son fundamentalmente por ampliar su formación $(47,2 \%)$, mientras el $29 \%$ lo hace por razones de su trabajo, el 13,4 por facilitar su ocio, el 7,9 porque le gustan los idiomas y solamente el $2,4 \%$ para poder viajar o para no olvidar lo que ya conoce.

\subsection{Instrumento}

En cuanto a la organización de las estrategias del cuestionario está basada en la clasificación de estrategias de aprendizaje de Franco (2007). Para su elaboración se han tenido en cuenta las taxonomías en la literatura consultada (O’Malley y Chamot, 1990; Hisiao y Oxford, 2002). Igualmente, se ha tomado en consideración la propuesta del marco común europeo de referencia para las lenguas (MCERL) (Council of Europe, 2001; Mecd, 2002).

Por tanto, se ha optado por clasificar las estrategias de aprendizaje en cuatro grandes grupos denominados estrategias metacognitivas, cognitivas, afectivas y sociales que se definen de la siguiente forma:

* Estrategias metacognitivas: son aquellas que sirven para dirigir el propio aprendizaje a través de su planificación, organización y evaluación.

* Estrategias cognitivas: son aquellas que intervienen en el procesamiento de la información $\mathrm{y}$, en concreto, en la comprensión y expresión de la lengua objeto de estudio.

* Estrategias afectivas: son las relacionadas con las creencias, las actitudes y la motivación.

* Estrategias sociales: son las que intervienen en la interacción con los demás.

A partir de este marco conceptual se realizó la definición de las diferentes categorías en función del tipo de estrategias y los ítems en cada una de ellas. Para evaluar la validez de contenido se convocó a tres expertos en elaboración de cuestionarios y a tres expertos en estrategias de aprendizaje en diferentes idiomas. Se realizaron dos reuniones con el 
fin de discutir la formulación de las preguntas y la representación de las diferentes dimensiones o categorías. Las reuniones, de 2 a 3 horas de duración, tuvieron lugar en la facultad de Ciencias de la Educación de la UNED en Madrid.

Se realizó también un pre-test a un grupo de alumnos de la Facultad de Educación de la Universidad de Vigo que cursaban la materia de inglés para conocer la duración de la entrevista y valorar la comprensión de los ítems.

El cuestionario de modalidad de aplicación colectiva, está compuesto de ocho preguntas de identificación, (Centro, nivel, asistencia a tutorías, sexo, edad, profesión, estudios, razones para el estudio de una lengua extranjera), 58 ítems cerrados con preguntas dicotómicas de SI y NO que nos permite saber el porcentaje de individuos que utiliza una determinada estrategia y una escala tipo Likert (58 ítems) que nos indica la frecuencia de uso de estrategias, en la cual 1 es nunca, 2 casi nunca, 3 algunas veces, 4 casi siempre y 5 siempre.

Por tanto, se ha optado por clasificar las estrategias de aprendizaje en cuatro grandes grupos denominados estrategias metacognitivas (16 ítems por cada variable), cognitivas (26 ítems por cada variable), afectivas (8 ítems) y sociales (8 ítems).

La interpretación de los datos se puede realizar de dos maneras. Por un lado, las preguntas con respuestas sí o no nos permiten saber el porcentaje de individuos que utiliza cada estrategia. De esta forma se averigua cuáles son las estrategias que más se utilizan siendo este dato más objetivo que el del la frecuencia de uso de las estrategias. En este sentido, este cuestionario permite obtener datos más objetivos que producirán resultados más fiables en la aplicación de las técnicas de inferencia estadística.

Por otro lado, empleamos en este cuestionario también el grado de frecuencia para matizar el sí o el no, sabiendo que estos datos expresan la percepción o la creencia del alumno y es más subjetivo. En la frecuencia existe un cierto grado de subjetividad. No obstante, es muy útil para matizar la respuesta de sí o no. Para la interpretación de los datos se establecen tres categorías de uso de las estrategias: a) un uso bajo que corresponde a las puntuaciones 1 y 2 ; b) un uso medio, que corresponde a la puntuación $3 \mathrm{y} ; \mathrm{c})$ un uso alto para las puntuaciones 4 y 5.

\subsection{Procedimiento}

Dada la dificultad de acceso al alumnado al tratarse de una enseñanza no presencial, se eligieron aquellos alumnos de los cuales disponíamos el e-mail y se procedió a convocarles en cada centro para explicarles la importancia del estudio y los objetivos que se pretendían alcanzar e informarles que en todo momento estaba garantizado el anonimato, la participación voluntaria y la confidencialidad de la información. Dado el tamaño del instrumento también se les explicó que se les enviaría por e-mail y que se tomaran su tiempo para contestar todo el instrumento. El propio programa una vez finalizado el cuestionario reenvía a las investigadoras el instrumento cubierto.

El requisito para formar parte de la muestra era solamente estar matriculado en el curso de inglés en el CUID.

La investigadora fue siempre la misma intentando mantener la misma actitud y motivación en todos los centros. Para ello se realizó un protocolo de actuación y respuestas a las posibles dudas presentadas por el alumnado y se realizaron ensayos previos con un grupo que no participaba en el estudio. 
El objetivo de este estudio es diseñar, aplicar y validar un instrumento para la evaluación de estrategias de aprendizaje en la enseñanza del inglés como lengua extranjera. Por ello se procede a los cálculos de fiabilidad y a analizar las interrelaciones entre los datos mediante el proceso del análisis factorial, es decir describir el número de variables o categorías en función de las cuales puede describirse la ejecución de cada individuo.

Ante la posibilidad de que un ítem obtenga saturaciones mayores de .30 en más de un componente será asignado a aquel cuyo coeficiente sea más alto (Camacho, 1995).

\section{Resultados}

\subsection{Análisis de ítems}

El análisis de los ítems se llevo a cabo mediante el índice de homogeneidad, calculado mediante la correlación producto-momento de Pearson entre la puntuación del ítem y la total de la escala. También se llevo a cabo la pertinente corrección (I.H.C) para neutralizar la contribución del ítem a la puntuación total, ya que sin esta corrección el índice de homogeneidad resultaría sobreestimado.

En la siguiente tabla se puede comprobar que todos los ítems fueron significativamente homogéneos, con un margen de error de uno por mil, lo cual nos indica que todos ellos están evaluando la misma variable.

Tabla 2. Índice de homogeneidad corregido

\begin{tabular}{|l|l|l|l|l|l|}
\hline Ítem & I.H.C & Ítem & I.H.C & Ítem & I.H.C \\
\hline 1 & .56 & 21 & .37 & 41 & .34 \\
\hline 2 & .53 & 22 & .37 & 42 & .53 \\
\hline 3 & .45 & 23 & .46 & 43 & .23 \\
\hline 4 & .56 & 24 & .51 & 44 & .34 \\
\hline 5 & .46 & 25 & .53 & 45 & .52 \\
\hline 6 & .34 & 26 & .56 & 46 & .50 \\
\hline 7 & .60 & 27 & .47 & 47 & .47 \\
\hline 8 & .36 & 28 & .57 & 48 & .37 \\
\hline 9 & .46 & 29 & .54 & 49 & .44 \\
\hline 10 & .56 & 30 & .53 & 50 & .45 \\
\hline 11 & .49 & 31 & .49 & 51 & .60 \\
\hline 12 & .49 & 32 & .49 & 52 & .56 \\
\hline 13 & .55 & 33 & .45 & 53 & .34 \\
\hline 14 & .45 & 34 & .39 & 54 & .34 \\
\hline 15 & .43 & 35 & .46 & 55 & .39 \\
\hline 16 & .47 & 36 & .36 & 56 & .45 \\
\hline 17 & .62 & 37 & .29 & 57 & .49 \\
\hline 18 & .59 & 38 & .33 & 58 & .52 \\
\hline 19 & .46 & 39 & .37 & & \\
\hline 20 & .38 & 40 & .43 & & \\
\hline
\end{tabular}


En la tabla podemos observar que todos los ítems fueron significativamente homogéneos con un margen de error del uno por mil, lo cual nos indica que todos ellos están evaluando la misma variable $\mathrm{y}$, por lo tanto, podemos considerar que resultan fiables.

\subsection{Análisis de la escala}

La fiabilidad se ha calculado mediante el cálculo de la consistencia interna (coeficiente alpha de Cronbach). Sus valores hallados para cada sexo, edad y nivel académico se pueden comprobar en la siguiente tabla.

Tabla 3. Medición de la Consistencia interna de la escala

\begin{tabular}{|l|l|}
\hline & Alpha de Cronbach \\
\hline Sexo & $\mathrm{H}=954$ \\
& $\mathrm{M}=0.943$ \\
\hline Edad & $>40$ años $=0.957$ \\
& $<40$ años $=0.946$ \\
\hline Nivel & Licenciado $=0.950$ \\
académico & $\begin{array}{l}\text { Diplomado }=0.942 \\
\text { Bachillerato }=0.949 \\
\end{array}$ \\
& Formación profesional $=0.950$ \\
& Doctor $=0.954$ \\
\hline
\end{tabular}

Con respecto a la muestra total se obtiene un valor de alpha 0.945 .

Como podemos observar los valores no defieren demasiado en función de las distintas variables lo que nos permite afirmar que nos encontramos con una escala con suficientes garantías de fiabilidad para ambos sexos, para las diferentes edades y nivel académico tenidas en cuenta en nuestro estudio.

Con el objetivo de analizar la validez de constructo del cuestionario se realizó un análisis factorial de componentes principales con posterior rotación VARIMAX. El índice de Kaiser-Meyer-Olkin (KMO) es de 0.728. Los valores bajos en este estadístico implican que las correlaciones entre cada pareja de variables no puede explicarse por otras y en este caso no podría utilizarse el análisis factorial. En nuestro caso esta cifra se aproxima a la unidad lo que significa que existen factores comunes.

La prueba de la esfericidad también resultó significativa $(\mathrm{p}=.000)$ por lo que entendemos que el análisis factorial es adecuado y el modelo consigue un buen ajuste.

El determinante obtenido de la correlación es de 0.069 lo que nos indica una alta presencia de correlaciones y, por lo tanto podemos confirmar de nuevo el ajuste del modelo factorial.

Recogemos para el análisis solamente las saturaciones superiores a 0.60 . Identificamos según este criterio cuatro factores, que explican $50,68 \%$ de la varianza de los datos (Ver tabla 4). 
Tabla 4. Estructura factorial de la escala

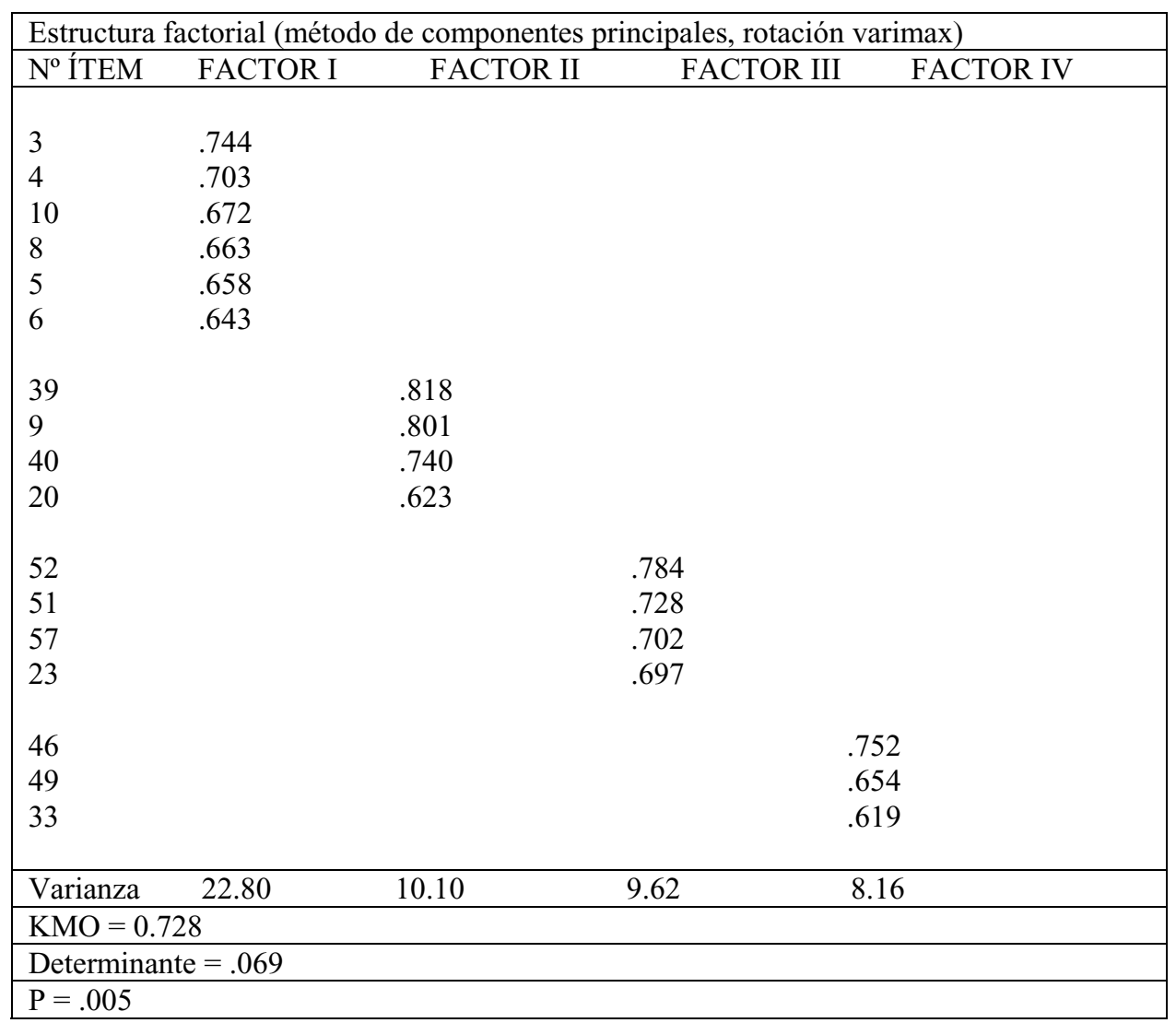

Fundamentalmente los ítems se agrupan en los mismos factores que ha sido dividida la escala. El primer componente, estrategias metacognitivas, explica el $22.80 \%$ de la varianza y está compuesto por 6 ítems, que hacen referencia a actividades de autoregulación del propio aprendizaje, es decir de planificar qué estrategias se han de utilizar en cada situación, aplicarlas, controlar el proceso, evaluarlo para detectar posibles fallos, y como consecuencia transferir todo ello a una nueva. Queda patente que un componente explica un porcentaje mucho más elevado de la varianza. En este factor saturan más de la mitad de los ítems aunque nosotros hemos seleccionado para el análisis los más altos.

Los ítems que tienen una saturación más alta en este componente se centran en planificación y dirección del aprendizaje centrándose en actividades como prestar atención concretándose en aspectos concretos, organizar un horario, averiguar mecanismos de aprendizaje y estudiar y practicar aspectos como gramática, vocabulario y pronunciación.

El ítem 6 destaca casi por igual en el componente 1 (estrategias metacognitivas) que el componente 2 (estrategias cognitivas). 
El segundo componente, que explica el $10.10 \%$ de la varianza se compone de 5 ítems, los cuales se relacionan con las estrategias cognitivas entendiendo estas como la organización de procesos mentales estructurados y orientados a conseguir un aprendizaje exitoso de forma rápida y eficaz. Los ítems se centran tanto en la práctica con medios audiovisuales como con libros de texto y en escuchar. Aparecen aquí dos ítems que nosotros habíamos clasificado dentro de las estrategias metacognitivas como son el ya comentado ítem 6 y el ítem 9 que se refiere a la utilización de técnicas de estudio adecuadas al contenido que se trabaja.

El tercer componente, que explica el $9.62 \%$ de la varianza, se configura con 4 ítems, relacionados con estrategias de tipo social relacionadas con las creencias, las actitudes y motivaciones. Los ítems que saturan más alto se refieren a solicitar que se aclaren palabras que no se entienden, que se repitan cuestiones que no se comprenden e interesarse por la cultura del país cuya lengua se está estudiando. Aquí también aparece un ítem saturando este factor que nosotros habíamos descrito dentro de las estrategias cognitivas (ítem 23) que se refiere al empleo de palabras nuevas en oraciones o situaciones. Podría tener una explicación al entenderse que esta tarea la haces fundamentalmente con otras personas.

El cuarto componente explica el $8.16 \%$ de la varianza y se configura con 3 ítems relacionados con estrategias afectivas que intervienen en la interacción con los demás. Los ítems seleccionados se refieren a la capacidad de tener pensamientos positivos para animarse a practicar la nueva lengua (ítem 46), buscar oportunidades para practicar la lengua con actividades divertidas y motivantes (ítem 49) y repasar los contenidos aprendidos (ítem 33). Este último ítem se había introducido en la escala dentro de las estrategias cognitivas.

\section{Conclusiones y discusión}

Existe una literatura bastante amplia sobre la importancia de las estrategias de aprendizaje en el dominio de una lengua extranjera. Sin embargo, existe gran escasez de estudios sobre las características de fiabilidad y validez de diferentes instrumentos de medida (Bailey, Onwuegbuzie \& Daley, 2000; Brown, 2000; Johnson, Prior \& Artuso, 2000; Stenberg \& Grigorenko, 2001; Ehrman y Leaver, 2003; Khalill, 2005; Jie y Xiaoqing, 2006; Demirbas y Demirkan, 2007; Choi, Lee y Jung, 2008; Lincoln y Rademacher, 2006). De ahí la importancia de este estudio.

La clasificación de las estrategias de aprendizaje en nuestro cuestionario tiene en cuenta, por tanto, los procesos de aprendizaje, lo que permite al profesor orientar al alumno en cómo utilizarlas para entender, asimilar y practicar con la lengua objeto de estudio. Asimismo, la organización del cuestionario está pensada para que el investigador pueda interpretar los resultados a la vista de qué estrategias emplean alumnos en los procesos de aprendizaje. La aplicación de las diversas técnicas estadísticas a los datos obtenidos permite comprobar si hay diferencias significativas entre el uso de las estrategias en dichos procesos y de esta forma obtener conclusiones objetivas al respecto. En esta línea, para la organización interna de cada grupo de estrategias propuesto se han 
tenido en cuenta los procesos que intervienen en el procesamiento de la información y en la enseñanza-aprendizaje de la siguiente forma:

Las estrategias metacognitivas intervienen en los procesos de planificación, dirección y evaluación. Suponen procesos ejecutivos de planeamiento para aprender, monitoreo de la propia comprensión y producción, y evaluación de cuán bien se ha logrado el objetivo de aprendizaje.

Las estrategias cognitivas forman parte de los procesos de procesamiento, asimilación y uso en las cuales el estudiante interactúa con el material a aprender manipulándolo mentalmente (por ejemplo, elaborando imágenes mentales, o trabajando sobre conceptos o habilidades adquiridas antes) o físicamente (por ejemplo, agrupando los elementos a aprender en categorías coherentes, o tomando notas de la información importante que debe recordarse).

Las estrategias afectivas desempeñan un papel importante en relación con el afecto, la motivación y la actitud. El alumno puede usar algún tipo de control afectivo para ayudarse en su tarea de aprendizaje.

Las sociales, que además de intervenir en aspectos como el afecto, la motivación y la actitud, están relacionadas con la cooperación y la empatía. El estudiante puede interactuar con otra persona para ayudarse en su aprendizaje -cooperando mutuamente, o haciendo preguntas para tener mayor claridad conceptual.

Debemos tener en cuenta que en este aspecto las ventajas de aprender de forma consciente e inconsciente para obtener un mayor éxito (Brown, 2002: 12).

Podemos concluir por el análisis realizado que los ítems de la escala presentan suficientes pruebas de homogeneidad en la muestra que se ha realizado. La escala de estrategias de aprendizaje del inglés como lengua extranjera puede considerarse suficientemente fiable para su utilización en una población de estudiantes adulta.

La puntuación obtenida por la escala nos permite conocer el grado de utilización de las diferentes estrategias de aprendizaje en el ámbito del dominio de una lengua extranjera.

Asimismo, la clasificación de estrategias en la que se basa el cuestionario no sólo ha servido para el desarrollo de este instrumento de investigación sino que también ha sido aplicada para tomar decisiones sobre cómo integrar las estrategias de aprendizaje en el currículo de las Escuelas Oficiales de Andalucía (BOJA 14-11-2007). En esta línea, es igualmente de utilidad como referente para el desarrollo de las programaciones departamentales y, por tanto, para su aplicación en las enseñanzas en el contexto del presente estudio, además de poder ser transferible a otros ámbitos educativos. De esta forma, se abren nuevas líneas de aplicación de la enseñanza estratégica en contextos educativos diferentes que desarrollen el marco común europeo de referencia para las lenguas.

\section{REFERENCIAS}

Anderson J.R. (1981). Cognitive skills and their acquisition. Hillsdale: N.J. Erlbaum Anderson J.R. (1985). The architecture of cognition. Cambridge MA.: Harward University Press. Bailey, P., Onwuegbuzie, A. J., \& Daley, C. E. (2000). "Using learning style to predict foreign language achievement at college level", System, 28, 115-133. 
Beltrán, J. (1996). Procesos, Estrategias y Técnicas de Aprendizaje. Madrid: Síntesis.

Bernard, J.A. (1999). Estrategias de Aprendizaje. Madrid: Bruño.

Brown, H. D. (2000). Principles of language learning and teaching. New York: Longman

Brown, H. D. (2002). Strategies for successs: A practical guide to learning English. CA: Prentice Hall.

Bruton, A. (1998). "Language learning strategies in FL pedagogy: sequences", in E. Alcon, y Codina V. (Eds.). Current Issues in English Language Methodology. Castellón: Servicio de Publicaciones de la Universitat Jaume I, 91-99.

Camacho, J. (1995). Análisis multivariado con SPSS/PC+. Barcelona: EUB.

Choi, I., Lee, S. J., \& Jung, J. W. (2008). "Designing Multimedia Case-Based Instruction Accommodating Students' Diverse Learning Styles", in Journal of Educational Multimedia and Hypermedia, 17, 1:5-25.

Cohen, A. D. (1998). Strategies in Learning and Using a Second Language. London: Longman.

Cohen, A. D., Oxford, R. y Chi, J.C. (2003). Language Strategy Use Inventory. CARLA working paper. Minneapolis, MN: Center for advanced research on language acquisition. http:// www.carla.umn.edu/about/whatsnew.html, accesed 9 december, 2010.

Cohen, A.D. (1987). "Studying learner strategies: how we get the information», in A. Wenden, y Rubin J. (1987). (eds.), Learner Strategies in Language Learning. Hemel Hempstead: Prentice Hall.

Cohen, A.D. y Macaro, E. (eds)(2007). Language Learner Strategies. Oxford: Oxford University Press.

Council of Europe (2001). Common European Framework of Reference for Languages: Learning, Teaching, Assessment. Strasbourg: Council of Europe.

Demirbas, O., y Demirkan, H. (2007). "Learning Styles of Design Students and the Relationship of Academic Performance and Gender in Design Education", in Learning and Instruction, 17, 3: 345-359.

Ehrman, M. E. y Leaver, B. L. (2003). "Cognitive styles in the service of language learning", in System, 31: 391-415.

Fernández Carril, R. (2004). "Vocabulary learning strategies and foreign language teaching", in M. Díez, R Fernández, y Halbach A. (eds.), Debating Learning Strategies, Frankfurt am Main: Stroemfeld Verlag, 193-201.

Fodor, J. (1983). The Modularity of the Mind. Cambridge: MIT Press.

Franco, P. (2007). "Estrategias de enseñanza-aprendizaje del inglés como lengua extranjera: diseño y aplicación de un modelo instruccional en el contexto de las Escuelas Oficiales de Idiomas". Tesis doctoral no publicada. Cádiz: Universidad de Cádiz.

Gagné, E. (1985). The Cognitive Psychology of School Learning. Boston: Little Brown and Company.

Hsiao, T.Y. y Oxford, R. L. (2002). "Comparing theories of language learning strategies: A confirmatory factor analysis", in Modern Language Journal, 86, 3: 368-383.

Hunt, M. (1982). The Universe Within: A New Science Explores the Human Mind. New York: Simon \& Schuster.

Jie, L., y Xiaoqing, Q. (2006). "Language Learning Styles And Learning Strategies Of Tertiarylevel English Learners In China", in RELC, 37, 1: 67-70.

Johnson, J., Prior, S., y Artuso, M. (2000). "Field dependence as a factor in second language communicative production", in Language learning, 50, 3: 529-567.

Khalil, A. (2005). "Assessment of Language Learning Strategies Used by Palestinian EFL Learners", in Foreign Language Annals, 38, 1: 108-119. 
Lincoln, F., y Rademacher, B. (2006). "The Learning Styles of ESL Students in Community Colleges", in Community College Journal of Research and Practice, 30: 485-500.

MECD (2002). Marco común europeo de referencia para las lenguas: aprendizaje, enseñanza, evaluación. Madrid, Ministerio de Educación, Ciencia y Deporte y Grupo Anaya S.A.

Nakatani, Y. (2005). "The effects of awareness-raising training on oral communication strategy use", in Modern Language Journal, 89, 1: 76-91.

O’Malley, J.M. y A.U. Chamot (1990). Learning Strategies in Second Language Acquisition. Cambridge: C.U.P.

ORDEN de 18 de octubre de 2007, por la que se desarrolla el currículo correspondiente a las enseñanzas de idiomas de régimen especial en Andalucía (BOJA 14-11-2007).

Oxford, R. (1990). Language Learning Strategies. Boston: Heinle \& Heinle.

Oxford, R. (1996). Employing a Questionnaire to Assess the use of language learning Strategies", in Applied language learning, 7, 1-2: 25-45.

Oxford, R. y Burry-Stock, J.A. (1995). "Assessing the use of language learning strategies WorldWide with the ESL/EFL Version of the strategy Inventory for Language Learning (SILL)", in System, 23, 1: 1-23.

Paige, R. M., Cohen, A. D., \& Shively, R. (2004). "Assessing the Impact of a Strategies-based Curriculum on Language and Culture Learning Abroad", in Frontiers: The Interdisciplinary Journal of Study Abroad, 10: 253-276.

Schmidt, R. (1990). "The role of consciousness in second language learning", in Applied Linguistics, 1, 2: $129-158$.

Schumann, J.H. (1994). "Where is cognition? Emotion and cognition in second language acquisition", in Studies in Second Language Acquisition, 16: 231-42.

Sheorey, R. y Mokhtari, K. (2001). "Differences in the metacognitive awareness of reading strategies among native and non-native readers" in System, 29: 431-449.

Skehan, P. (1998). A cognitive approach to language learning. Oxford: Oxford University Press.

Sternberg, R. J., \& Grigorenko, E. L. (2001). "A capsule history of theory and research on styles". in R. J. Sternberg \& L. F. Zhang (Eds.), Perspectives on thinking, learning and cognitive styles. Mahwah, NJ: Lawrence Erlbaum Associates, 1-21.

Valcarcel, M.; Coyle, Y. y Verdú, M. (1996). "Learning foreign languages: learner strategies", in McLaren, D. y Madrid, D. (eds). A Handbook for TEFL. Alicante: Marfil

Vandergrift, L.; Goh, C.C.M.; Mareschal, C. y Taflaghodtari, M.H. (2006). The metacognitive awareness Listening Questionnaire: developement and validation", in Language Learning, 56, 3: 431-462.

VanPatten, B. (1990). "Attending to form and content in the input: an experiment in consciousness", in Studies in second language acquisition, 12: 287-301.

VanPatten, B. (1996). Input processing and grammar instruction in second language acquisition. Norwood, NJ: Ablex Publishing Corporation.

Wenden, A. (1991). Learner Strategies for Learner Autonomy. Cambridge: Prentice Hall.

White, C., Schramm, K. y Chamot, A.U. (2007). "Research methods in strategy research: reexamining the toolbox", in A. Cohen and E. Macaro (Eds.), Language learner strategies: 30 years of research and practice. Oxford: Oxford University Press, 93-116. 


\section{ApÉNDICE}

ESCALA

\begin{tabular}{|c|c|}
\hline PARTE A & \begin{tabular}{|l|l|l}
$N^{\circ}$ \\
Ítem
\end{tabular} \\
\hline Antes de hablar o escribir sobre un tema, ¿te paras a pensar sobre él? & 1 \\
\hline $\begin{array}{l}\text { Antes de hablar o escribir sobre un tema, ¿organizas tus ideas adecuadamente } \\
\text { decidiendo cómo vas a empezar y a terminar la exposición? }\end{array}$ & 2 \\
\hline $\begin{array}{l}\text { Antes de una tarea de aprendizaje, ¿decides prestar atención a lo que vas a hacer } \\
\text { evitando distraerte? }\end{array}$ & 3 \\
\hline $\begin{array}{l}\text { Antes de una tarea de aprendizaje, ¿decides prestar atención a aspectos concretos } \\
\text { que te ayudaría a hacerla como el vocabulario, las palabras claves o la } \\
\text { pronunciación? }\end{array}$ & 4 \\
\hline $\begin{array}{l}\text { ¿Estudias y practicas la gramática, el vocabulario y la pronunciación para aprender } \\
\text { a entender la lengua de estudio y a comunicarte? }\end{array}$ & 5 \\
\hline $\begin{array}{l}\text { ¿Conoces y utilizas términos para hablar o entender la gramática u otros aspectos } \\
\text { de la lengua cuando los estudias o en las actividades que realizas? }\end{array}$ & 6 \\
\hline $\begin{array}{l}\text { ¿Te propones objetivos según tus necesidades para mejorar en el uso de lengua de } \\
\text { estudio? }\end{array}$ & 7 \\
\hline ¿Tratas de averiguar cómo aprender mejor la lengua de estudio? & 8 \\
\hline $\begin{array}{l}\text { ¿Utilizas estrategias, técnicas de estudio o de trabajo adecuadas para ti o para los } \\
\text { contenidos y destrezas que vas a trabajar? }\end{array}$ & 9 \\
\hline $\begin{array}{l}\text { ¿Organizas tu horario personal para tener tiempo y poder aprender la lengua de } \\
\text { estudio? }\end{array}$ & 10 \\
\hline ¿Organizas y utilizas adecuadamente el material de aprendizaje? & 11 \\
\hline $\begin{array}{l}\text { ¿Buscas oportunidades fuera del aula para practicar la lengua leyendo, escuchando, } \\
\text { escribiendo o hablando con otras personas? }\end{array}$ & 12 \\
\hline $\begin{array}{l}\text { ¿Buscas oportunidades de utilizar recursos fuera del aula para aprender la lengua } \\
\text { de estudio? }\end{array}$ & 13 \\
\hline ¿Te das cuentas de los errores mientras realizas la tarea en la lengua de estudio? & 14 \\
\hline ¿Intentas darte cuenta y aprender de los errores para hacerlo mejor? & 15 \\
\hline ¿Intentas corregir las tareas por ti mismo? & 16 \\
\hline \multicolumn{2}{|l|}{ PARTE B } \\
\hline $\begin{array}{l}\text { ¿Prestas atención a la información general y/o concreta cuando escuchas, lees, } \\
\text { hablas o escribes en la lengua de estudio? }\end{array}$ & 17 \\
\hline $\begin{array}{l}\text { ¿Utilizas la información que sabes para predecir respuestas o adivinar el } \\
\text { significado cuando no conoces alguna palabra? }\end{array}$ & 18 \\
\hline $\begin{array}{l}\text { ¿Empleas materiales como gramáticas, libros de ejercicios e internet entre otros } \\
\text { para aprender la lengua de estudio? }\end{array}$ & 19 \\
\hline ¿Escuchas la lengua de estudio y la imitas y repites oralmente para recordarla? & 20 \\
\hline ¿Lees la lengua de estudio y la escribes varias veces para recordarla? & 21 \\
\hline ¿Traduces palabra por palabra para entender lo que escuchas o lees? & 22 \\
\hline ¿Empleas palabras nuevas en oraciones o situaciones? & 23 \\
\hline ¿Usas ejemplos para comprender y recordar la lengua de estudio? & 24 \\
\hline ¿Clasificas las palabras según el tema u otros criterios en tablas o esquemas? & 25 \\
\hline ¿Analizas las palabras, frases o textos para comprenderlos? & 26 \\
\hline ¿Intentas analizar estructuras y crear reglas? & 27 \\
\hline ¿Aprendes las reglas y las aplicas? & 28 \\
\hline
\end{tabular}




\begin{tabular}{|c|c|}
\hline ¿Utilizas el subrayado? & 29 \\
\hline ¿Tomas notas? & 30 \\
\hline ¿Reelaboras las notas que tomas para expresar su contenido? & 31 \\
\hline ¿Empleas el resumen? & 32 \\
\hline ¿Repasas los contenidos que has aprendido? & 33 \\
\hline ¿Relacionas lo que ya sabes con lo nuevo que aprendes? & 34 \\
\hline ¿Ensayas antes de realizar una tarea? & 35 \\
\hline Cuando no te acuerdas de una palabra concreta, ¿utilizas gestos? & 36 \\
\hline Cuando no te acuerdas de una palabra concreta, ¿te la inventas? & 37 \\
\hline $\begin{array}{l}\text { Cuando no te acuerdas de una palabra concreta, ¿empleas una palabra o frase que } \\
\text { signifique lo mismo? }\end{array}$ & 38 \\
\hline ¿Practicas la lengua aprendida viendo películas o programas de televisión? & 39 \\
\hline ¿Practicas la lengua aprendida leyendo revistas o libros de tu interés? & 40 \\
\hline $\begin{array}{l}\text { ¿Practicas la lengua aprendida escribiendo mensajes de correo electrónico o cartas } \\
\text { para comunicarte con otras personas fuera de clase? }\end{array}$ & 41 \\
\hline ¿Practicas la lengua aprendida hablando con otras personas fuera de clase? & 42 \\
\hline \multicolumn{2}{|l|}{ PARTE C } \\
\hline ¿Notas que estás tenso o nervioso cuando tienes que realizar una tarea? & 43 \\
\hline ¿Cuándo sientes nerviosismo al realizar una tarea, intentas relajarte? & 44 \\
\hline Cuando no entiendes algo ¿intentas no bloquearte y continuar con la tarea? & 45 \\
\hline ¿Tienes pensamientos positivos para animarte a practicar la lengua de estudio? & 46 \\
\hline $\begin{array}{l}\text { ¿Comentas con otras personas cómo te sientes cuando practicas la lengua de } \\
\text { estudio? }\end{array}$ & 47 \\
\hline ¿Te das algún premio cuando realizas bien una tarea? & 48 \\
\hline $\begin{array}{l}\text { ¿Intentas buscar oportunidades para practicar la lengua con actividades que te } \\
\text { divierten y motivan a seguir aprendiendo? }\end{array}$ & 49 \\
\hline $\begin{array}{l}\text { ¿Te das cuenta de cómo tus emociones, creencias o actitudes afectan tu aprendizaje } \\
\text { e intentas que te ayuden a mejorar? }\end{array}$ & 50 \\
\hline \multicolumn{2}{|l|}{ PARTE D } \\
\hline Cuando no entiendes algo, ¿pides que te lo digan más despacio o que te lo repitan? & 51 \\
\hline ¿Pides que te aclaren o confirmen con otras palabras lo que no entiendes? & 52 \\
\hline ¿Trabajas con otros compañeros de estudio para aprender la lengua? & 53 \\
\hline ¿Pides a hablantes nativos que te ayuden a aprender? & 54 \\
\hline ¿Pides a los demás que te corrijan? & 55 \\
\hline ¿Intercambias tus opiniones y sentimientos con los demás en la lengua de estudio? & 56 \\
\hline ¿Te interesa la cultura del país o países donde se habla la lengua? & 57 \\
\hline ¿Realizas alguna actividad de ocio en la lengua de estudio? & 58 \\
\hline
\end{tabular}

